\title{
Dynamics of Ion Transport in Ionic Liquids
}

\author{
Alpha A. Lee, ${ }^{1}$ Svyatoslav Kondrat, ${ }^{2,3}$ Dominic Vella, ${ }^{1}$ and Alain Goriely ${ }^{1}$ \\ ${ }^{1}$ Mathematical Institute, Andrew Wiles Building, University of Oxford, Woodstock Road, \\ Oxford OX2 6GG, United Kingdom \\ ${ }^{2}$ IBG-1: Biotechnology, Forschungszentrum Jülich, 52425 Jülich, Germany \\ ${ }^{3}$ Department of Chemistry, Faculty of Natural Sciences, Imperial College London, \\ London SW7 2AZ, United Kingdom
}

(Received 4 February 2015; revised manuscript received 7 July 2015; published 4 September 2015)

\begin{abstract}
A gap in understanding the link between continuum theories of ion transport in ionic liquids and the underlying microscopic dynamics has hindered the development of frameworks for transport phenomena in these concentrated electrolytes. Here, we construct a continuum theory for ion transport in ionic liquids by coarse graining a simple exclusion process of interacting particles on a lattice. The resulting dynamical equations can be written as a gradient flow with a mobility matrix that vanishes at high densities. This form of the mobility matrix gives rise to a charging behavior that is different to the one known for electrolytic solutions, but which agrees qualitatively with the phenomenology observed in experiments and simulations.
\end{abstract}

DOI: 10.1103/PhysRevLett.115.106101

Room temperature ionic liquids play an increasingly important role as electrolytes in electrochemical and electromechanical applications ranging from actuators [1-3] to supercapacitors [4-8]. Ionic liquids differ from traditional electrolytes in that they consist only of positive and negative ions without any solvent. Recent theoretical calculations [9] suggest that ionic liquids are concentrated electrolytes, and should not be modeled as a weak electrolyte (with solvent consisting of ion pairs), as suggested elsewhere [10].

In many important technological applications, ionic liquids are close to an electrified interface [11]. Although the equilibrium structure of the electrical double layer is relatively well studied, understanding the dynamic response of ionic liquids to an applied potential or surface charge is more challenging because of the difficulty in identifying an appropriate nonequilibrium dynamic framework. Previous theoretical studies [12-17] relied on the dynamical density functional theory $[18,19]$, in which the ion flux $\mathbf{j}_{ \pm}$is related to the ion density fields $c_{ \pm}$and free energy density functional $F\left[c_{ \pm}\right]$via

$$
\mathbf{j}_{ \pm}=-M_{ \pm} c_{ \pm} \nabla\left(\frac{\delta F}{\delta c_{ \pm}}\right)
$$

where $M_{ \pm}$is the cation or anion mobility. A key assumption in the derivation of Eq. (1) is that ion density is low compared to an underlying solvent bath [20]. This assumption may become problematic, as can be illustrated by substituting the one-component lattice gas free energy, $\mathcal{F}_{\text {ex }}=\left(k_{B} T /\right.$ $\left.a^{3}\right) \int_{\Omega}\left[a^{3} c \times \ln \left(a^{3} c\right)+\left(1-a^{3} c\right) \ln \left(1-a^{3} c\right)\right] d^{3} x$, where $c$ is the particle density and $\Omega$ is the system's volume, into Eq. (1); applying the continuity equation, we obtain

$$
\frac{\partial c}{\partial t}=D \nabla \cdot\left(\frac{\nabla c}{1-a^{3} c}\right)
$$

PACS numbers: 82.45.Gj, 47.57.jd, 78.30.cd, 82.45.Fk

where $D=M k_{B} T$ is the diffusion constant, $k_{B}$ the Boltzmann constant, and $T$ temperature. However, the continuum limit of a system of particles on a lattice with lattice constant $a$ undergoing a simple exclusion process is well known, and leads to the linear diffusion equation $\partial c / \partial t=D \nabla^{2} c$ [21], rather than Eq. (2).

Lattice gas models of ions, first proposed by Bikerman [22] in the 1940s, are commonly used as simple models of ionic liquids $[12,13,23-25]$ in equilibrium. The goal of this Letter is to derive a consistent model for the dynamics of ions in solvent-free ionic liquids, and analyze the dynamics of electrical double layer formation. We map the system onto a lattice and take the continuum limit of the microscopic reference kinetics of a discrete symmetric exclusion process (see Fig. 1). This reference kinetics is a natural one to consider as ion motion in a concentrated assembly is physically akin to particles "hopping" on a lattice [26]. Similar reference kinetics were successfully used to model

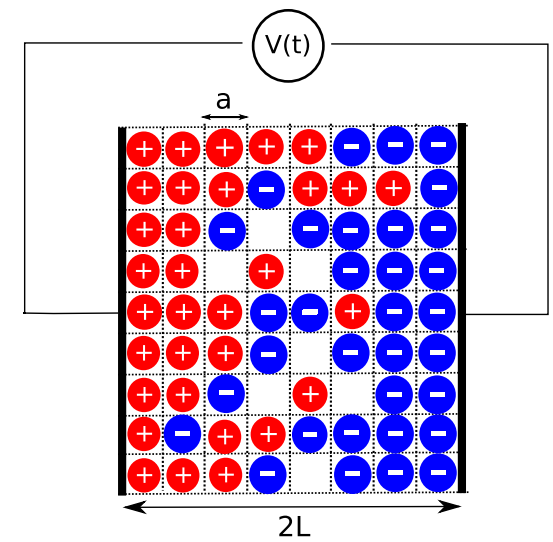

FIG. 1 (color online). Schematic of the system under consideration: cations and anions on a lattice of lattice constant $a$. 
spinodal decomposition in alloys [29-33], and were shown to be a microscopic basis for the Cahn-Hilliard equation [34-36]. Our approach has the advantage that steric exclusion is accounted for at the level of dynamics.

We first consider a one-dimensional lattice of lattice constant $a$ (corresponding to ion diameter $a$ in the continuum limit) for simplicity, and later generalize our results to higher dimensions. We consider a discrete-time dynamics in which particles can only move between nearest-neighbor lattice sites between time $t$ and $t+\Delta t$. Denoting by $S_{i}^{\alpha}(t) \in\{0,1\}$ the occupancy of the $i$ th lattice site at time $t$ by an ion of type $\alpha=\{+,-\}$, the evolution master equation for $S_{i}^{\alpha}$ reads

$$
\begin{aligned}
S_{i}^{\alpha}(t+\Delta t)= & r_{i \rightarrow i+1}^{\alpha} S_{i}^{\alpha} S_{i+1}+r_{i+1 \rightarrow i}^{\alpha} S_{i+1}^{\alpha}\left(1-S_{i}\right) \\
& +r_{i \rightarrow i-1}^{\alpha} S_{i}^{\alpha} S_{i-1}+r_{i-1 \rightarrow i}^{\alpha} S_{i-1}^{\alpha}\left(1-S_{i}\right) \\
& +\left(1-r_{i \rightarrow i+1}^{\alpha}-r_{i \rightarrow i-1}^{\alpha}\right) S_{i}^{\alpha},
\end{aligned}
$$

where the $S_{i}^{\alpha}$ on the right-hand side are taken at time $t$ and $S_{i}=S_{i}^{+}+S_{i}^{-} ; r_{i \rightarrow i \pm 1}^{\alpha}$ is unity if particle $\alpha$ at site $i$ attempts to jump to site $i \pm 1$ and is zero otherwise: this transition propensity takes into account the long-ranged interparticle interactions as explained below. The first term of Eq. (3) ensures that there will be particle $\alpha$ at site $i$ and time $t+\Delta t$, if it is there at time $t$, attempts to move to site $i+1$ and finds that site occupied. The second term describes a possible transition from site $i+1$ to site $i$ when there is no particle at site $i$. The third and forth terms describe the same processes between sites $i$ and $i-1$. Finally, the last term corresponds to a particle $\alpha$ at site $i$ that does not attempt to leave it during the interval $\Delta t$.

An ensemble average of $r_{i \rightarrow j}^{\alpha}$ with $|i-j|=1$ gives a transition rate that satisfies the detailed balance and can thus be related to the Boltzmann factor by

$$
\left\langle r_{i \rightarrow j}^{\alpha}\right\rangle=\frac{1}{2} e^{-\left(V_{j}^{\alpha}-V_{i}^{\alpha}\right) / 2 k_{B} T}
$$

where

$$
V_{i}^{\alpha}=\sum_{j \neq i} \sum_{\beta} U_{\alpha \beta}(|i-j|) S_{j}^{\beta}
$$

is a potential acting on particle $\alpha$ at site $i$ due to all remaining particles, and $U_{\alpha \beta}(|i-j|)$ is the microscopic (electrostatic) interaction potential between the particles. In the absence of long-ranged interactions $\left(U_{\alpha \beta}=0\right)$, there is no preferred direction of motion, thus $\left\langle r_{i \rightarrow i \pm 1}^{\alpha}\right\rangle=1 / 2$.

The continuum evolution equation can be obtained by rescaling the lattice indices by the lattice spacing $a$, and introducing the minimal lattice volume $v(v=a$ in 1D) as well as the ensemble average concentrations $c_{\alpha}(x=a i)=$ $v^{-1}\left\langle S_{i}^{\alpha}\right\rangle$ and mean potentials $\mu_{\alpha}^{l}(x)=\sum_{\beta} \int_{\Omega} U_{\alpha \beta}(\mid x-$ $\left.x^{\prime} \mid\right) c_{\beta}\left(x^{\prime}\right) d x^{\prime}$. Taking the average of both sides of Eq. (3), and applying the mean field approximation, $\left\langle S_{i}^{\alpha} S_{j}^{\beta} \ldots\right\rangle \approx\left\langle S_{i}^{\alpha}\right\rangle\left\langle S_{j}^{\beta}\right\rangle \ldots$, we can expand the resulting expression in a power series in $a$ and $\Delta t$ to obtain

$$
\begin{aligned}
\frac{1}{D} \frac{\partial c_{\alpha}}{\partial t}= & v c_{\alpha} \frac{\partial^{2} c}{\partial x^{2}}+(1-v c) \frac{\partial^{2} c_{\alpha}}{\partial x^{2}}+c_{\alpha}(1-v c) \frac{1}{k_{B} T} \frac{\partial^{2} \mu_{\alpha}^{l}}{\partial x^{2}} \\
& -\left[v c_{\alpha} \frac{\partial c}{\partial x}-(1-v c) \frac{\partial c_{\alpha}}{\partial x}\right] \frac{1}{k_{B} T} \frac{\partial \mu_{\alpha}^{l}}{\partial x}
\end{aligned}
$$

where we have defined $c=c_{+}+c_{-}$as the total ion density, and identified $D=a^{2} /(2 \Delta t)$ as the self-diffusion coefficient.

To generalize Eq. (6) to higher dimensions, we assume that the fluxes along different axes are decoupled. Introducing the mobility matrix

$$
\mathcal{M}=\frac{D}{k_{B} T}\left(\begin{array}{cc}
c_{+}(1-v c) & 0 \\
0 & c_{-}(1-v c)
\end{array}\right),
$$

the higher-dimensional version of (6) is

$$
\frac{\partial \boldsymbol{c}}{\partial t}=\nabla \cdot(\mathcal{M} \nabla \boldsymbol{\mu})
$$

where $\boldsymbol{c}^{T}=\left(c_{+}, c_{-}\right), \boldsymbol{\mu}^{T}=\left(\mu_{+}, \mu_{-}\right)$, and $\mu_{ \pm}$is defined by $\mu_{ \pm}=\delta \mathcal{F} / \delta c_{ \pm}$, where

$$
\begin{aligned}
\mathcal{F}\left[c_{ \pm}\right]= & \frac{1}{2} \sum_{\alpha \beta} \int_{\Omega} c_{\alpha}\left(\mathbf{x}_{1}\right) U_{\alpha \beta}\left(\left|\mathbf{x}_{1}-\mathbf{x}_{2}\right|\right) c_{\beta}\left(\mathbf{x}_{2}\right) d \mathbf{x}_{1} d \mathbf{x}_{2} \\
& +\frac{k_{B} T}{v} \int_{\Omega}\left[\sum_{\alpha} v c_{\alpha} \ln \left(v c_{\alpha}\right)\right. \\
& +(1-v c) \ln (1-v c)] d \mathbf{x} .
\end{aligned}
$$

Equation (8) is the continuum kinetic equation for an interacting two component system. Note the important physical constraint that the evolution equation for the total concentration, $c$, in the absence of long-ranged interactions $\left(U_{\alpha \beta}=0\right)$, reduces to the linear diffusion equation. This constraint, as explained above, respects the fact that the underlying dynamics of our reference system is a simple exclusion process on a lattice. Continuum kinetic equations with the same mobility function as Eq. (8) have been proposed in the literature in the context of the modified Cahn-Hilliard equation [37], and phase-field models of Li-ion batteries [38,39].

To apply Eq. (8) to an ionic liquid system, we introduce a characteristic length scale $l_{c}$ for short-ranged interactions, and split the Coulomb potential $U_{\alpha \beta}=U_{\alpha \beta}^{s r}+U_{\alpha \beta}^{l r}$, where $U_{\alpha \beta}^{s r}(x)=q_{\alpha} q_{\beta} l_{B} e^{-x / l_{c}} / x, U_{\alpha \beta}^{l r}(x)=q_{\alpha} q_{\beta} l_{B}\left(1-e^{-x / l_{c}}\right) / x$ [40,41], and $l_{B}=e^{2} /\left(4 \pi \epsilon_{0} \epsilon k_{B} T\right)$ is the Bjerrum length. Below the length scale $l_{c}$, it is actually the hard core exclusion that matters rather than the Coulomb interaction; thus, $U_{\alpha \beta}^{s r}$ can be neglected. This truncation of the 


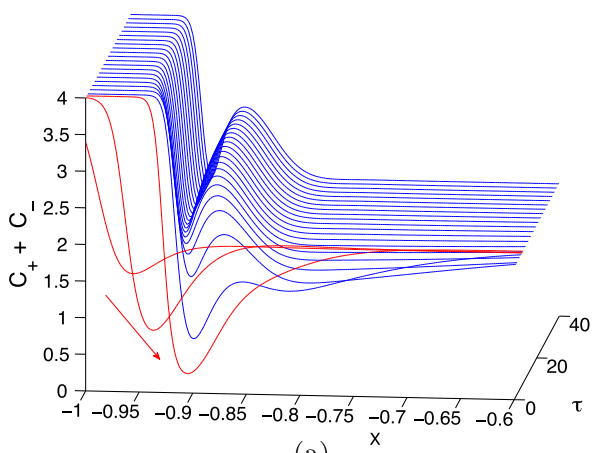

(a)

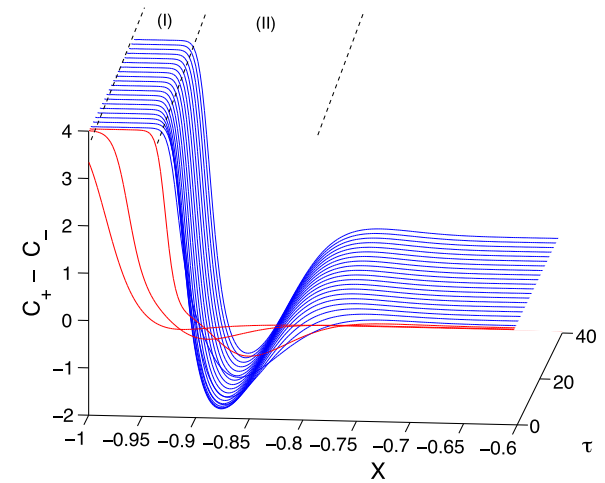

(b)

FIG. 2 (color online). (a) The total density, $C_{+}+C_{-}$, and (b) charge density $C_{+}-C_{-}$, as functions of distance $X=x / L$ from the electrode and time after an applied step voltage $V=$ $40 V_{T}$ with $V_{T}=k_{B} T / e$. Here, $\gamma=0.25, l_{c} / l_{D}=10$, and $L / l_{D}=100$, red and blue curves denote the first and second charging regimes, and the arrow shows the increasing density deficit in the first charging regime. Numerical solution of Eqs. (11)-(12) is performed using PDEPE in Matlab.

Coulomb potential is necessary as our mean-field approach underestimates steric correlations, and as such the divergence of the Coulomb interaction at the origin renders electrostatic interactions effectively too strong. We thus write

$$
\frac{U_{\alpha \beta}(x)}{k_{B} T} \approx \frac{U_{\alpha \beta}^{l r}(x)}{k_{B} T}=q_{\alpha} q_{\beta} l_{B} \frac{1-e^{-x / l_{c}}}{x} .
$$

This decomposition of the Coulomb potential is not unique-the exponential function is chosen phenomenologically and for mathematical convenience.

Introducing the local electric field $u$, and exploiting the Green function, one can rewrite the nonlocal integrodifferential equation (8) as a set of coupled partial differential equations

$$
\begin{gathered}
\left(1-l_{c}^{2} \nabla^{2}\right) \nabla^{2} u=-4 \pi l_{B}\left(c_{+}-c_{-}\right), \\
\frac{\partial c_{ \pm}}{\partial t}=D \nabla \cdot c_{ \pm}(1-v c) \nabla\left[ \pm u+\ln \left(\frac{v c_{ \pm}}{1-v c}\right)\right] .
\end{gathered}
$$

Equation (11) is identical to the modified Poisson equation derived phenomenologically in Ref. [25] using a gradient expansion of a nonlocal electrostatic kernel. We note that Ref. [42] took the variational approach of Ref. [25] to develop a framework for charge-transfer reaction kinetics, with the resulting equation similar to Eq. (12). Here we provided a microscopic statistical derivation of the kinetics of ion transport.

We turn our attention to a simple problem to gain some insight into the characteristic behavior of Eqs. (11)-(12): an ionic liquid with bulk cation and anion concentration $c_{0}$ bounded by two parallel, blocking electrodes at $x=-L, L$. Initially the concentrations of the two ion species are uniform, and a step voltage of amplitude $2 V$ is applied at $t=0^{+}$. Introducing the Debye length $l_{D}=1 / \sqrt{8 \pi c_{0} l_{B}}$ and dimensionless packing parameter in the bulk [12,23] $\gamma=v c_{0}$, we introduce the dimensionless variables $\tau=$ $\left(D / L l_{D}\right) t, X=x / L, C_{ \pm}=c_{ \pm} / c_{0}$. The no-flux conditions at the electrodes read

$$
\left[ \pm C_{ \pm}(1-\gamma C) \frac{\partial u}{\partial X}+(1-\gamma C) \frac{\partial C_{ \pm}}{\partial X}+\gamma C_{ \pm} \frac{\partial C}{\partial X}\right]_{X= \pm 1}=0
$$

At the electrodes surface, we posit that the classical Gauss law $\pm \epsilon u_{x}=4 \pi \sigma$ holds at $X= \pm 1$, with $\sigma$ the (dimensional) surface charge density, and $\epsilon$ the dielectric constant of the medium $[25,43]$. This condition, together with the constant potential condition gives

$u(X= \pm 1, \tau)= \pm V, \quad u_{X X X}(X= \pm 1, \tau)=0, \tau>0$.

The initial conditions are

$$
C_{ \pm}(X, 0)=1, X \in[-1,1] .
$$

To avoid complications of double layer overlap, we consider widely separated electrodes taking $L / l_{D}=100$. We take $l_{c}=a=v^{1 / 3}$, Bjerrum length $l_{B}=50 \AA$, ion diameter $a=5 \AA$, and $\gamma=0.25$ (see, e.g., Ref. [25] though the qualitative behavior reported below is not sensitive to $\gamma$ ); we therefore have $l_{c} / l_{D}=\sqrt{8 \pi \gamma l_{B} / a} \approx 10$.

Figure 2 shows that charging proceeds through two distinct regimes: First, the (negative) electrode attracts cations from the vicinity and expels anions, resulting in a dense, "compact layer" of cations near the electrode that overcompensates the surface charge (region I in Fig. 2(b)). Ion diffusion is hindered as the mobility matrix (7) vanishes in regions of high density. As a result, the total density reaches a minimum away from the compact layer [cf. red arrow in Fig. 2(a)]. In the second stage, anions arrive from the bulk to screen the now net-positive compact layer. This flux fills the total density deficit near the compact layer incurred in the first charging regime, creating a region of 


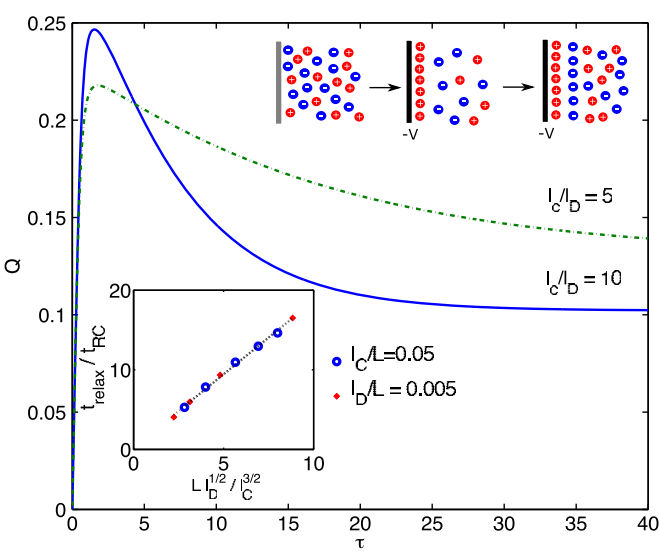

FIG. 3 (color online). The total charge as a function of time for different values of $l_{c} / l_{D}$ with $L / l_{D}=100, \gamma=0.25$ fixed. (Inset) The equilibrium relaxation time $\tau_{\text {relax }}$ as a function of $l_{c}$ and $l_{D}$ (obtained by fitting numerical results to an exponential decay).

negative charge density and, in fact, excess total density [region II in Fig. 2(b)].

A key measure of practical interest is the integrated total diffuse charge,

$$
Q(\tau)=\int_{-1}^{0}\left[C_{+}(X, \tau)-C_{-}(X, \tau)\right] d X .
$$

Note that the overall system is electroneutral; therefore, the total charge of the ions is equal and opposite to the surface charge. $Q$ is therefore the charge accumulated at the anode, which is equal and opposite in sign to the charge accumulated at the cathode. Figure 3 shows that, as charging proceeds, the total charge initially increases, corresponding to the formation of the compact layer. However, arrival of anions in the second charging regime decreases the charge to the final equilibrium value. This charging mechanism is schematically illustrated in the inset of Fig. 3. The correlation length $l_{c} / l_{D}$ controls the extent of charge oscillation and thus of overcompensation of electrode surface charge by the compact layer. Therefore, decreasing the correlation length reduces the extent of charge overcompensation and also the peak diffuse charge.

Further insights into the charging process can be obtained by noting that the initial rise in charge occurs over $\tau=O(1)$. In dimensional terms this corresponds to $t_{R C}=L l_{D} / D$, the usual $R C$ time constant [44], corroborating the fact that the peak has its origin in the formation of the diffuse layer. Numerical experimentation (see inset of Fig. 3) suggests that the late-stage exponential relaxation of the charge to equilibrium has a distinctly different time scale

$$
t_{\text {relax }}=\frac{L^{2}}{D}\left(\frac{l_{D}}{l_{c}}\right)^{3 / 2}
$$

This scaling suggests that the decay in the stored charge comes from the formation of charge oscillations: $L^{2} / D$ gives

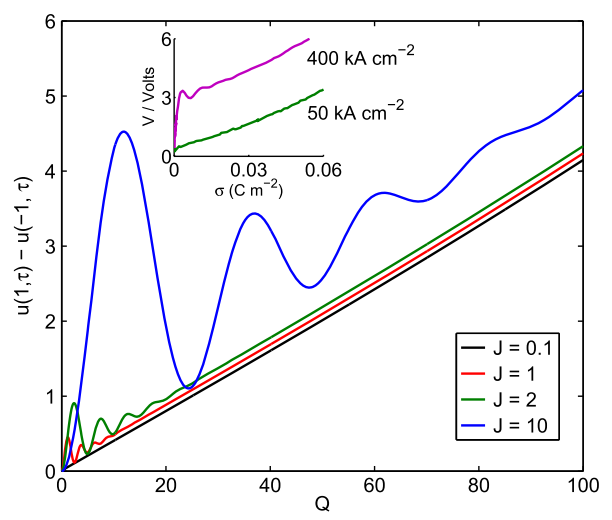

FIG. 4 (color online). The voltage drop across the system evolves nonmonotonically under constant current conditions, Eq. (18). Here $L / l_{D}=100, l_{c} / l_{D}=10$, and $\gamma=0.25$, and the total charge $Q=J \tau$. Inset: simulation data from Ref. [45] for which $\approx 400 \mathrm{kA} \mathrm{cm}^{-2}$ corresponds to dimensionless $J=2$.

the decay time due to diffusion of ions through the electrochemical cell, and this is rescaled by $\left(l_{D} / l_{c}\right)^{3 / 2}$, where $l_{c}$ is the characteristic wavelength of charge oscillations [cf. Eq. (11)].

The nonmontonic evolution of $Q(t)$ is in stark contrast to the results predicted by dynamical density functional theory $[13,16,17]$, where the diffuse charge is monotonically increasing. We note that this effect is different from kinetic charge inversion due to double layer overlap [15]. The degenerate mobility (7) in our approach ensures that the flux due to electrostatic interactions vanishes at close packing, and thus there are distinct regimes of initial charge density polarization and, at later times, rearrangement of the double layer into cation-rich and anion-rich layers.

Qualitatively similar behavior is obtained under chargecontrolled conditions, i.e., imposing a constant current,

$$
\left.\frac{\partial u}{\partial X}\right|_{X= \pm 1}= \pm J \tau
$$

Figure 4 shows that the nonequilibrium double layer rearrangement manifests itself in the nonmonotonic evolution of the potential drop across the system when the current density $J$ is large. This qualitatively agrees with recent molecular dynamics simulations [45], but is in contrast to conventional dynamical density functional theory, which again predicts a monotonic increase in potential drop as a function of time.

In summary, we have derived a continuous model for the dynamics of solvent-free ionic liquids based on coarsegraining a simple exclusion process of interacting particles defined on a lattice. The resulting equations have the structure of a gradient flow with a degenerate mobility function. As examples, these equations were analyzed for a system where (i) a step voltage is applied between widely separated electrodes, and (ii) a constant charging current is applied. Even in these simple cases, our theory differs 
qualitatively from previously developed theories for electrolyte solutions. Importantly, we showed that the total diffuse charge is a nonmonotonic function of time. Experiments and simulations of the dynamics of ion transport in ionic liquids are currently scarce; we hope that our theory provides a framework to interpret experiments and motivate further investigation.

We thank S. Perkin for discussions about the structure of ionic liquids, and G. Oshanin and A. A. Kornyshev for discussions about kinetic lattice gas systems. This work is supported by an EPSRC Research Studentship to A. A. L. A. G acknowledges support from a Wolfson/ Royal Society Merit Award. All data accompanying this publication are directly available within the publication.

[1] S. Nemat-Nasser, Springer Handbook of Experimental Solid Mechanics (Springer, New York, 2008), p. 187.

[2] S. Liu, W. Liu, Y. Liu, J.-H. Lin, X. Zhou, M. J. Janik, R. H. Colby, and Q. Zhang, Polym. Int. 59, 321 (2010).

[3] A. A. Lee, R. H. Colby, and A. A. Kornyshev, Soft Matter 9, 3767 (2013).

[4] J. Chmiola, G. Yushin, Y. Gogotsi, C. Portet, P. Simon, and P. L. Taberna, Science 313, 1760 (2006).

[5] C. Largeot, C. Portet, J. Chmiola, P. Taberna, Y. Gogotsi, and P. Simon, J. Am. Chem. Soc. 130, 2730 (2008).

[6] S. Kondrat and A. Kornyshev, J. Phys. Condens. Matter 23, 022201 (2011).

[7] P. Simon and Y. Gogotsi, Acc. Chem. Res. 46, 1094 (2013).

[8] A. A. Lee, S. Kondrat, and A. A. Kornyshev, Phys. Rev. Lett. 113, 048701 (2014).

[9] A. A. Lee, D. Vella, S. Perkin, and A. Goriely, J. Phys. Chem. Lett. 6, 159 (2015).

[10] M. A. Gebbie, M. Valtiner, X. Banquy, E. T. Fox, W. A. Henderson, and J. N. Israelachvili, Proc. Natl. Acad. Sci. U.S.A. 110, 9674 (2013).

[11] M. V. Fedorov and A. A. Kornyshev, Chem. Rev. 114, 2978 (2014).

[12] M. S. Kilic, M. Z. Bazant, and A. Ajdari, Phys. Rev. E 75, 021503 (2007).

[13] H. Zhao, Phys. Rev. E 84, 051504 (2011).

[14] J. Jiang, D. Cao, D.-e. Jiang, and J. Wu, J. Phys. Condens. Matter 26, 284102 (2014).

[15] J. Jiang, D. Cao, De-en Jiang, and J. Wu, J. Phys. Chem. Lett. 5, 2195 (2014).

[16] A. Yochelis, Phys. Chem. Chem. Phys. 16, 2836 (2014).

[17] A. Yochelis, J. Phys. Chem. C 118, 5716 (2014).

[18] U. M. B. Marconi and P. Tarazona, J. Chem. Phys. 110, 8032 (1999).
[19] U. M. B. Marconi and P. Tarazona, J. Phys. Condens. Matter 12, A413 (2000)

[20] P. Español and H. Löwen, J. Chem. Phys. 131, 244101 (2009).

[21] H. Spohn, Large Scale Dynamics of Interacting Particles (Springer, New York, 1991), Vol. 825.

[22] J. J. Bikerman, London, Edinburgh Dublin Philos. Mag. J. Sci. 33, 384 (1942).

[23] A. A. Kornyshev, J. Phys. Chem. B 111, 5545 (2007).

[24] M. Z. Bazant, M. S. Kilic, B. D. Storey, and A. Ajdari, Adv. Colloid Interface Sci. 152, 48 (2009).

[25] M. Z. Bazant, B. D. Storey, and A. A. Kornyshev, Phys. Rev. Lett. 106, 046102 (2011).

[26] Simulations show that the nanostructure of ionic liquids with long alkyl chains can be considered as a microphaseseparated network of polar and non-polar domains [27,28]. It is thus reasonable to assume that ions move by jumping between polar domains.

[27] J. N. Canongia Lopes and A. A. Pádua, J. Phys. Chem. B 110, 3330 (2006).

[28] L. M. Santos, J. N. Canongia Lopes, J. A. Coutinho, J. M. Esperança, L. R. Gomes, I. M. Marrucho, and L. P. Rebelo, J. Am. Chem. Soc. 129, 284 (2007).

[29] J.-F. Gouyet, Europhys. Lett. 21, 335 (1993).

[30] M. Plapp and J.-F. Gouyet, Phys. Rev. Lett. 78, 4970 (1997).

[31] M. Plapp and J.-F. Gouyet, Eur. Phys. J. B 9, 267 (1999).

[32] J.-F. Gouyet, M. Plapp, W. Dieterich, and P. Maass, Adv. Phys. 52, 523 (2003).

[33] E. Petrishcheva and R. Abart, Acta Mater. 60, 5481 (2012).

[34] G. Giacomin and J. L. Lebowitz, Phys. Rev. Lett. 76, 1094 (1996).

[35] G. Giacomin and J. L. Lebowitz, J. Stat. Phys. 87, 37 (1997).

[36] G. Giacomin and J. L. Lebowitz, SIAM J. Appl. Math. 58, 1707 (1998).

[37] E. B. Nauman and D. Q. He, Chem. Eng. Sci. 56, 1999 (2001).

[38] T. R. Ferguson and M. Z. Bazant, J. Electrochem. Soc. 159, A1967 (2012).

[39] Y. Zeng and M. Z. Bazant, SIAM J. Appl. Math. 74, 980 (2014).

[40] Y.-G. Chen and J. D. Weeks, Proc. Natl. Acad. Sci. U.S.A. 103, 7560 (2006).

[41] C. D. Santangelo, Phys. Rev. E 73, 041512 (2006).

[42] M. Z. Bazant, Acc. Chem. Res. 46, 1144 (2013).

[43] B. D. Storey and M. Z. Bazant, Phys. Rev. E 86, 056303 (2012).

[44] M. Z. Bazant, K. Thornton, and A. Ajdari, Phys. Rev. E 70, 021506 (2004).

[45] X. Jiang, J. Huang, H. Zhao, B. G. Sumpter, and R. Qiao, J. Phys. Condens. Matter 26, 284109 (2014). 